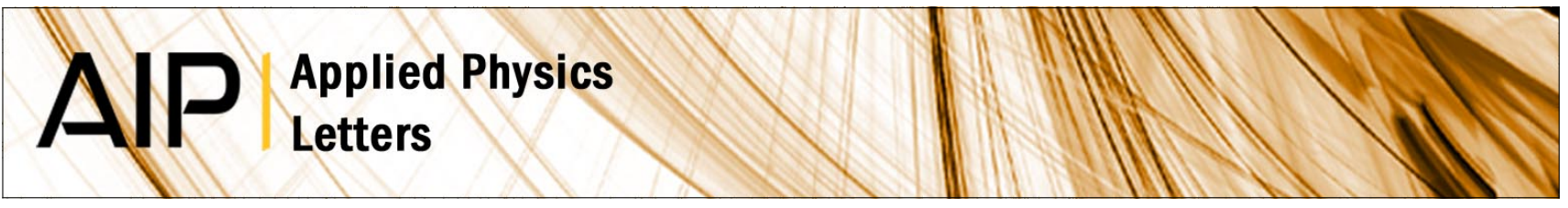

\title{
Nanoparticle-assisted microwave absorption by single-wall carbon nanotubes
}

A. Wadhawan, D. Garrett, and J. M. Perez

Citation: Appl. Phys. Lett. 83, 2683 (2003); doi: 10.1063/1.1615679

View online: http://dx.doi.org/10.1063/1.1615679

View Table of Contents: http://apl.aip.org/resource/1/APPLAB/v83/i13

Published by the American Institute of Physics.

\section{Related Articles}

Metal-assisted electroless fabrication of nanoporous $p-G a N$ for increasing the light extraction efficiency of light emitting diodes

AlP Advances 2, 012109 (2012)

Irradiance influence on the multicolor photochromism of mesoporous $\mathrm{TiO} 2$ films loaded with silver nanoparticles Appl. Phys. Lett. 99, 173106 (2011)

Computational study on structural modification of single-walled carbon nanotubes by electron irradiation J. Appl. Phys. 109, 054304 (2011)

Multiwalled carbon nanotubes and dispersed nanodiamond novel hybrids: Microscopic structure evolution, physical properties, and radiation resilience

J. Appl. Phys. 109, 014314 (2011)

Continuous-wave laser annealing of Si-rich oxide: A microscopic picture of macroscopic Si-SiO2 phase separation

J. Appl. Phys. 108, 124301 (2010)

\section{Additional information on Appl. Phys. Lett.}

Journal Homepage: http://apl.aip.org/

Journal Information: http://apl.aip.org/about/about_the_journal

Top downloads: http://apl.aip.org/features/most_downloaded

Information for Authors: http://apl.aip.org/authors

\section{ADVERTISEMENT}




\title{
Nanoparticle-assisted microwave absorption by single-wall carbon nanotubes
}

\author{
A. Wadhawan, D. Garrett, ${ }^{\text {a) }}$ and J. M. Perez ${ }^{\text {b) }}$ \\ Department of Physics, University of North Texas, Denton, Texas 76203
}

(Received 28 April 2003; accepted 5 August 2003)

\begin{abstract}
We report the effects of microwave irradiation on both unpurified and purified iron-catalyzed high-pressure disproportionation (HiPco)-grown single-walled carbon nanotubes (SWNTs) in ultrahigh vacuum. Under microwave irradiation, we observe that unpurified HiPco SWNTs quickly reach temperatures of approximately $1850^{\circ} \mathrm{C}$. As a result, $\mathrm{H}_{2}, \mathrm{H}_{2} \mathrm{O}, \mathrm{CO}, \mathrm{CO}_{2}$, and $\mathrm{CH}_{4}$ gases are observed, and the Fe catalyst nanoparticles melt and coalesce into larger crystallites approximately four times their original diameter. In contrast, carbon black and purified HiPco SWNTs heat up to temperatures of $500-650{ }^{\circ} \mathrm{C}$. We propose that the significant heating of unpurified HiPco SWNTs is due to the Fe catalysts. (C) 2003 American Institute of Physics. [DOI: 10.1063/1.1615679]
\end{abstract}

Carbon nanotubes have recently attracted considerable interest due to the unique mechanical and electronic properties of this material. ${ }^{1}$ However, the effects of microwave radiation on carbon nanotubes have not been extensively studied. We have recently reported preliminary studies on the absorption of microwave radiation by iron-catalyzed highpressure disproportionation (HiPco)-grown ${ }^{2,3}$ single wall carbon nanotubes (SWNTs). ${ }^{4}$ In this letter, we compare in detail the effects of microwave radiation on purified and unpurified HiPco SWNTs, and carbon black in ultrahigh vacuum (UHV). Under microwave irradiation, we observe that unpurified HiPco SWNTs quickly reach temperatures of approximately $1850^{\circ} \mathrm{C}$, glowing bright yellow in color. As a result, $\mathrm{H}_{2}, \mathrm{H}_{2} \mathrm{O}, \mathrm{CO}, \mathrm{CO}_{2}$, and $\mathrm{CH}_{4}$ gases are observed. The HiPco process utilizes $\mathrm{Fe}$ clusters as catalysts. ${ }^{2,3}$ Consequently, the as-grown unpurified material contains impurity Fe nanoparticles that are 3-5 $\mathrm{nm}$ in diameter and imbedded in carbon shells. Using transmission electron microscopy (TEM), we observe that after microwave irradiation the $\mathrm{Fe}$ nanoparticles have coalesced into large crystallites having an average diameter about four times larger than that of the original nanoparticles. In contrast, purified HiPco SWNTs that have significantly fewer catalyst impurities heat up to about $650^{\circ} \mathrm{C}$ and carbon black samples heat up to temperatures of about $500{ }^{\circ} \mathrm{C}$. We propose that the significant heating of unpurified HiPco SWNTs is due to the Fe catalysts.

Two varieties of HiPco SWNT bundles, unpurified and purified (BuckyPearls ${ }^{\mathrm{TM}}$ ), were purchased from Carbon Nanotechnologies, Inc. ${ }^{5}$ Both have an average SWNT diameter of approximately $1.1 \mathrm{~nm}$ with the unpurified and purified variants having an impurity Fe content of about 10.4 and 2.3 at. $\%$, respectively. The carbon black was made by thermal decomposition of acetylene and has an impurity content $<0.01 \mathrm{wt} \%$. Approximately $0.03 \mathrm{~g}$ of each material is placed in a Pyrex glass tube that is attached to an UHV system equipped with a Stanford Research Systems residual gas analyzer (RGA) model 200. The UHV system is baked out at

\footnotetext{
${ }^{a)}$ Department of Materials Science and Engineering, University of North Texas, Denton, Texas, 76203.

${ }^{b)}$ Electronic mail: jperez@unt.edu
}

$120^{\circ} \mathrm{C}$ for two days and pumped down to pressures below $10^{-10}$ Torr. The sample is sighted by an optical pyrometer and a high-resolution video camera. In addition, an Ocean Optics Spectrometer with a charge coupled device detector sensitive from $300 \mathrm{~nm}$ to $1.0 \mu \mathrm{m}$ is set up to take emission spectra. The microwave source utilized for these experiments was a $1000 \mathrm{~W}$ magnetron operating at a frequency of 2.45 GHz.

The magnetron was placed approximately $0.5 \mathrm{~cm}$ below the test tube, as shown in Fig. 1(a), and the samples irradiated for intervals of time less than $10.0 \mathrm{~s}$. As shown in Fig. 1(b), the unpurified HiPco SWNTs glowed yellow after less than $0.04 \mathrm{~s}$ of irradiation, which is the time interval between frames of the video camera. After approximately $4.0 \mathrm{~s}$ of irradiation, the unpurified HiPco SWNTs flashed purple colored light that clearly originated in a region of the glass tube located above the nanotube sample, as seen in Fig. 1(c). We attribute this to a hydrogen plasma, which is under further study. The temperature of the glowing nanotubes shown in Fig. 1(b) was measured using a disappearing filament pyrometer to be $1850{ }^{\circ} \mathrm{C}$, assuming an emissivity of 1.0 . Light emission spectra from the glowing nanotubes obtained using the spectrometer were broadband and showed no peaks. There is production of gases with the primary gas observed being $\mathrm{H}_{2}$. RGA analysis also shows an increase of other gases such as $\mathrm{H}_{2} \mathrm{O}, \mathrm{CO}, \mathrm{CO}_{2}$, and $\mathrm{CH}_{4}$. Since the nanotubes were not intentionally exposed to $\mathrm{H}_{2}$, the $\mathrm{H}_{2}$ observed is attributed to the decomposition of hydrogen containing compounds on the nanotubes at high temperatures, as previously reported. ${ }^{6,7}$ The large amount of $\mathrm{H}_{2}$ in our experiment is likely due to the high temperature of $1850{ }^{\circ} \mathrm{C}$ reached by the nanotubes.

The same procedure was used to study the effects of microwaves on purified HiPco SWNTs and carbon black. These materials reached temperatures of 650 and $500{ }^{\circ} \mathrm{C}$, respectively, after $10 \mathrm{~s}$ of irradiation. The purified samples did not glow but had a few small regions that intermittently sparkled. The density of the unpurified HiPco SWNTs is about $0.01 \mathrm{~g} / \mathrm{cc}$. Due to the purification process, the purified HiPco SWNTs have a density that is about 40 times greater than that of the unpurified HiPco SWNTs. ${ }^{5}$ In order to deter- 


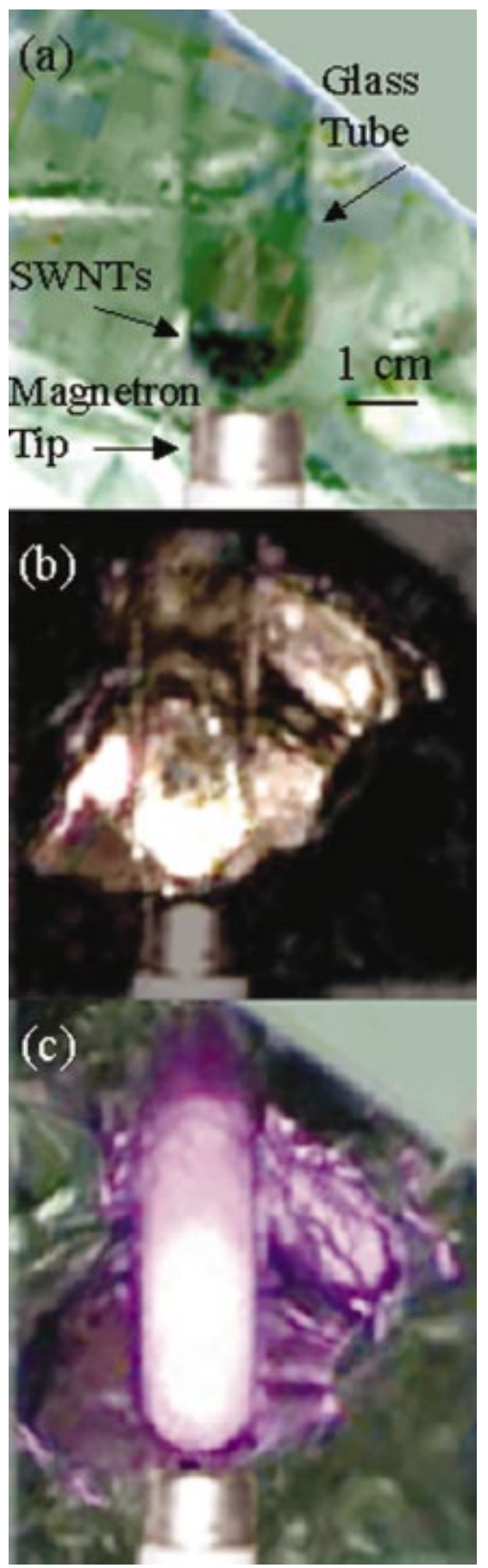

FIG. 1. (Color) Unpurified HiPco carbon nanotubes in a test tube evacuated to $<10^{-10}$ Torr under microwave irradiation at time durations, $t$. (a) Before microwave irradiation, (b) after $t=0.04 \mathrm{~s}$, and (c) after $t=4 \mathrm{~s}$.

mine if the lower density of unpurified HiPco SWNTs was responsible for the heating, we made mats of unpurified HiPco SWNTs having a density similar to that of the purified HiPco SWNTs by mixing the unpurified SWNTs in methanol and vacuum filtering the mixture. We found that the mats of unpurified HiPco SWNTs similarly reached temperatures of $1850^{\circ} \mathrm{C}$ and glowed bright yellow under irradiation, showing that density does not play a significant role.

Figures 2(a) and 2(b) are TEM micrographs of the unpurified HiPco SWNTs before and after $10 \mathrm{~s}$ of microwave irradiation. The arrow in Fig. 2(b) shows where large impurity structures, having an average diameter of about $18 \mathrm{~nm}$,
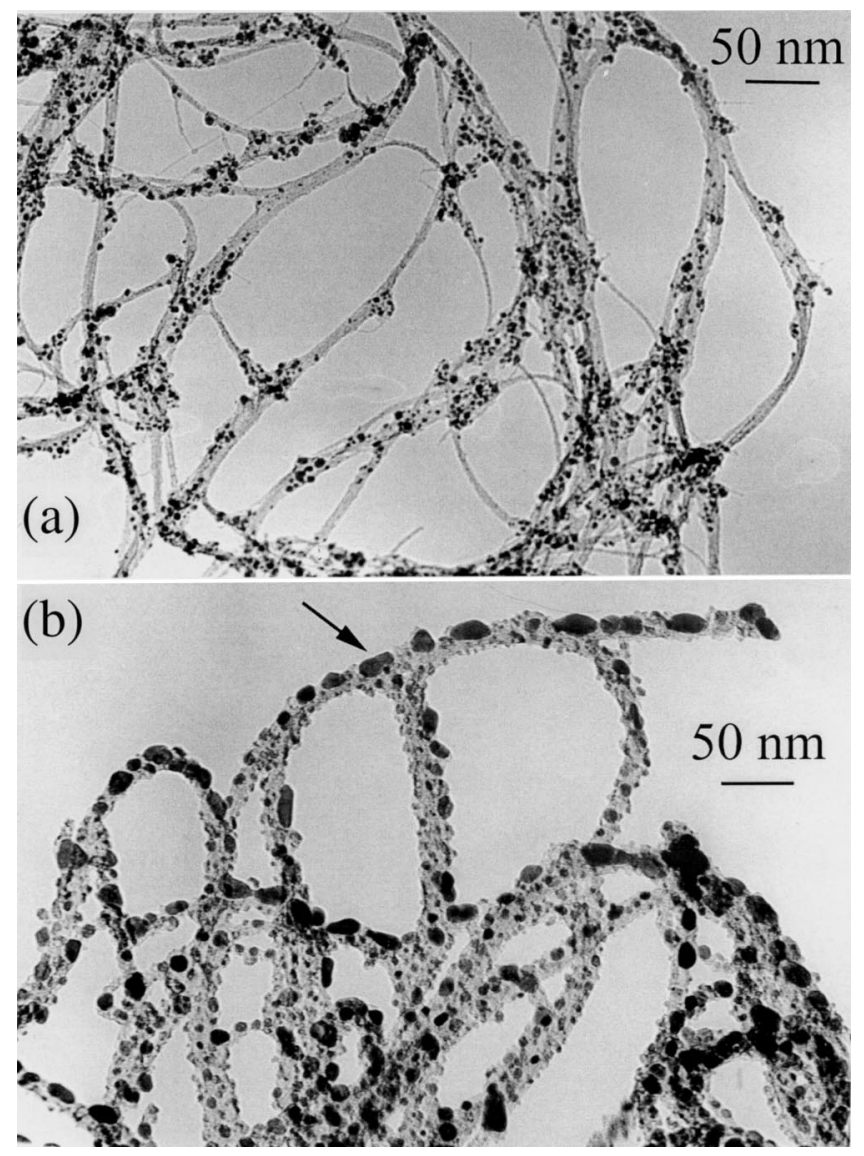

FIG. 2. TEM images showing (a) unpurified HiPco nanotubes before microwave irradiation, and (b) unpurified HiPco nanotubes after microwave irradiation. The Fe nanoparticles in (a) have coalesced into large crystallites, indicated by the arrow, having a diameter about four times greater than that of the original nanoparticles.

have crystallized on the outside walls of the SWNT bundles. Using energy-dispersive $\mathrm{x}$-ray spectroscopic analysis, the crystallites in Fig. 2(b) were found to be Fe. The SWNT bundles in Fig. 2(b) do not appear to be damaged although Raman spectra indicates diminishing of the radial breathing mode peaks at approximately 183 and $264 \mathrm{~cm}^{-1}$. In our setup, the SWNT sample is in an UHV and we believe the intense heating of the iron catalyst causes the catalyst to melt and break from the carbon shell. In the molten state, it migrates in search of "cold spots" where it coalesces and forms large amalgams of the impurity metal. Recently, a "nanowick" effect on the nanometer scale was reported in which metal catalysts in SWNTs were observed to melt and coalesce into larger particles when a nanometer-size current probe was used to locally heat the nanotubes. ${ }^{8}$ This effect was attributed to a temperature gradient. In all of the irradiated unpurified HiPco SWNT samples we studied, the coalescence of Fe catalysts was observed. We did not observe a significant increase in size of the Fe catalysts for the purified samples. Acid reflux treatments have been utilized to remove impurity nanoparticles from the unpurified HiPco SWNTs. ${ }^{3}$ It is likely that the acid reflux treatments will work more on the larger crystal aggregates formed after microwave irradiation leading to a more "purified" nanotube product.

A possible mechanism for the absorption of microwaves by SWNTs is adsorbed gas molecules. SWNTs utilized as gas sensors and exposed to $\mathrm{NH}_{3}$ for 10 min have shown 
slightly enhanced microwave absorption. ${ }^{9}$ Molecular dipoles formed on the nanotube surface and interstitial channels interact with the microwave field leading to some absorption losses through heating. However, we find that in our case gas adsorption is not a significant mechanism for the heating of HiPco SWNTs since the samples after considerable gas desorption continued to heat up as effectively.

Particulate matrices consisting of ferromagnetic particles imbedded in a network composite have attracted great interest as microwave radiation absorbing and shielding materials in the $\mathrm{GHz}$ frequency range. Examples of such structures include $\mathrm{Ba}$ hexaferrite nanocrystals ${ }^{10}$ and micron-size ferrite powders ${ }^{11,12}$ in a rubber matrix, $\mathrm{Fe}-\mathrm{Si}-\mathrm{Al}$ alloy flakespolymer composites, ${ }^{13}$ and CaCoTi ferrite-epoxy composites. ${ }^{14}$ Microwave absorption in these materials is thought to be mainly due to magnetic resonance and interfacial electric polarization. ${ }^{15,16}$ Magnetic resonance absorption occurs due to the coupling of the microwave field to the internal magnetic moments and is influenced by the shape of the particles. ${ }^{16}$ Interfacial polarization absorption occurs due to the interaction of microwave radiation with charge multipoles at the interface. ${ }^{15,16}$ For granular $\mathrm{Fe}-\mathrm{Ag}$ films, it has been reported that $\mathrm{Fe}$ nanoparticles having an average diameter greater than about $2.9 \mathrm{~nm}$ are ferromagnetic. ${ }^{17} \mathrm{HiPco}$ SWNTs contain Fe catalysts that have diameters of 3-5 nm and are unoxidized because they are imbedded in carbon shells. ${ }^{3}$ Therefore, we believe the Fe catalysts in HiPco SWNTs are ferromagnetic and absorb microwaves via magnetic resonance. The main example of interfacial dipoles in HiPco SWNTs is along the boundaries between the Fe catalyst particles and carbon shells. It has recently been reported that $\mathrm{Ba}$ hexaferrite crystallites in a rubber matrix absorb significantly more microwave radiation when the crystallites are nanometer-size instead of micron size. ${ }^{10}$ This effect is attributed to an increase in interfacial polarization as the crystallite size decreases due to an increase in the number of surface atoms with unsaturated bonds. ${ }^{10}$ For unpurified HiPco SWNTs, interfacial dipole absorption would be greater for the Fe catalyst nanoparticles imbedded in carbon shells than for the coalesced crytallites shown in Fig. 2(b) since the former are smaller and have greater interfacial polarization effects. In Ref. 10, it was also speculated that enhanced absorption in nanocrystals may be due to their quantum size which results in an increase in their energy level spacing. If the energy states lie in the range of the microwave energies, there could be microwave absorption due to electron transitions between the states. In addition, it is believed that since ferromagnetic nanoparticles have a single magnetic domain structure and higher coercive force, this may lead to hysteresis related losses. ${ }^{10}$

In summary, we have studied the effects of microwaves on unpurified and purified HiPco SWNTs and carbon black in an UHV. We find that unpurified HiPco SWNTs heat up to significantly higher temperatures than purified SWNTs and carbon black. This effect is attributed to greater microwave absorption due to the higher concentration of $\mathrm{Fe}$ catalyst nanoparticles. As a result of microwave irradiation, $\mathrm{H}_{2}$, $\mathrm{H}_{2} \mathrm{O}, \mathrm{CO}, \mathrm{CO}_{2}$, and $\mathrm{CH}_{4}$ are observed. The $\mathrm{Fe}$ catalyst nanoparticles present in the unpurified HiPco nanotubes melt and coalesce into crystallites approximately four times their original diameter.

The authors thank Jeff Day for the TEM images. This work was supported by the National Science Foundation under Award No. DMR-0074636, and the Texas Advanced Technology Program under Award No. 003594-0048-1999.

\footnotetext{
${ }^{1}$ See, for example, Physical Properties of Carbon Nanotubes, edited by R. Saito, G. Dresselhaus, and M. S. Dresselhaus (Imperial College Press, London, 1998).

${ }^{2}$ P. Nikolaev, M. J. Bronikowski, R. K. Bradley, F. Rohmund, D. T. Colbert, K. A. Smith, and R. E. Smalley, Chem. Phys. Lett. 313, 91 (1999).

${ }^{3}$ I. W. Chiang, B. E. Brinson, A. Y. Huang, P. A. Willis, M. J. Bronikowski, J. L. Margrave, R. E. Smalley, and R. H. Hauge, J. Phys. Chem. B 105, 8297 (2001).

${ }^{4}$ T. J. Imholt, C. A. Dyke, B. Hasslacher, J. M. Perez, D. W. Price, J. A. Roberts, J. B. Scott, A. Wadhawan, Z. Ye, and J. M. Tour, Chem. Mater. (to be published),

${ }^{5}$ Carbon Nanotehnologies, Inc., Houston, TX 77084 (www.cnanotech.com).

${ }^{6}$ A. Kuznetsova, D. B. Mawhinney, V. Naumenko, J. T. Yates, J. Liu, and R. E. Smalley, Chem. Phys. Lett. 321, 292 (2000).

${ }^{7}$ A. Kuznetsova, J. T. Yates, J. Liu, and R. E. Smalley, J. Chem. Phys. 112, 9590 (2000)

${ }^{8}$ B. C. Regan, S. Aloni, and A. Zettl, Bull. Am. Phys. Soc. 48, 813 (2003).

${ }^{9}$ S. Chopra, A. Pham, J. Gaillard, A. Parker, and A. M. Rao, Appl. Phys. Lett. 80, 4632 (2002).

${ }^{10}$ S. Ruan, B. Xu, H. Suo, F. Wu, S. Xiang, and M. Zhao, J. Magn. Magn. Mater. 212, 175 (2000).

${ }^{11}$ Y. Naito and K. Suetake, IEEE Trans. Microwave Theory Tech. 19, 65 (1971).

${ }^{12}$ S. Sugimoto, T. Maeda, D. Book, T. Kagotani, K. Inomata, M. Homma, H. Ota, Y. Houjou, and R. Sato, J. Alloys Compd. 330, 301 (2002).

${ }^{13}$ S. Yoshida, M. Sato, E. Sugawara, and Y. Shimada, J. Appl. Phys. 85, 4636 (1999).

${ }^{14}$ P. Singh, V. K. Babbar, A. Razdan, R. K. Puri, and T. C. Goel, J. Appl. Phys. 87, 4362 (2000).

${ }^{15}$ C. G. Koops, Phys. Rev. 83, 121 (1951).

${ }^{16}$ P. A. Miles, W. B. Westphal, and A. von Hippel, Rev. Mod. Phys. 29, 279 (1957).

${ }^{17}$ C. Peng, S. Zhang, G. Li, and D. Dai, J. Appl. Phys. 76, 998 (1994).
} 